\section{Epidemiología del cáncer de tiroides en un Instituto Oncológico. Efecto de las nuevas recomendaciones clínicas}

\author{
JORGE SAPUNAR Z. ${ }^{1,2, a}$, PEDRO FERRER R..$^{1, a}$
}

\section{Incidence of thyroid cancer in an oncological clinic of Santiago, Chile}

\begin{abstract}
Background: In Chile, Thyroid cancer (TC) is included in a list of diseases that have a guaranteed access to treatment, endorsed by the Ministry of Health. The care burden of the disease will depend on its incidence. Aim: To estimate the incidence of TC in Chilean patients with access to thyroid surgery and describe its histopathological characteristics. Material and methods: Analysis of the registry of all thyroidectomies performed at a private oncological clinic in Metropolitan Santiago, between 2016 and 2018. Incidence was estimated the number of persons affiliated to an insurance system managed by the same clinic, separated by age and gender. Results: TC adjusted incidence varied from 60.8 cases per 100,000 persons/year in 2016 to 48.7 cases per 100,000 persons/ year in 2018. The proportion of microtumors was 34\% when the diameter of all foci was considered. Papillary and follicular carcinomas were the pathological diagnoses in 96 and 2\% of cases respectively. Twenty-one percent of tumors had extra-thyroidal invasion. Conclusions: The TC incidence herein reported is higher than the figure of 7.9 cases per 100,000 persons/year reported by us in 2014. This difference could be due to a true increase in the incidence of TC, following worldwide trends or to differences in the methods used in both studies.

(Rev Med Chile 2020; 148: 573-581)
\end{abstract}

Key words: Epidemiology; Neoplasm Staging; Thyroid Neoplasms.
'Unidad de Investigación y Docencia, Instituto Oncológico Fundación Arturo López Pérez. ${ }^{2}$ Centro EPICYN y Departamento de Medicina Interna, Facultad de Medicina, Universidad de La Frontera. Temuco, Chile. ${ }^{\mathrm{a}} \mathrm{Mg}$.

Trabajo no recibió financiamiento. Los autores declaran no tener conflictos de interés.

Recibido el 26 de noviembre de 2019, aceptado el 5 de mayo de 2020 .

Correspondencia a: Jorge Sapunar Z.

Manuel Montt 112. Temuco,

Chile.

jorge.sapunar@ufrontera.cl
E $\mathrm{n}$ las últimas décadas se ha observado a nivel mundial un aumento progresivo en la incidencia del cáncer de tiroides (CT), con tasas muy variables en los distintos reportes ${ }^{1,2}$. Aunque este aumento parece depender principalmente de tumores pequeños del tipo histológico papilar ${ }^{1}$, el análisis de grandes registros de neoplasias también ha demostrado un aumento en la incidencia y mortalidad por cánceres avanzados ${ }^{3}$. Estos hallazgos han generado controversia respecto a la importancia epidemiológica del CT y de los posibles factores de riesgo involucrados ${ }^{1-3}$.

Recientemente las autoridades de salud de nuestro país decidieron incluir el cáncer diferenciado y medular del tiroides en personas de 15 años o más entre las enfermedades con atención garantizada ${ }^{4}$. Para poder estimar la carga asistencial que esta decisión producirá es necesario actualizar los datos de epidemiología del CT disponibles en Chile ${ }^{5,6}$.El CT, parece ser un hallazgo extremadamente frecuente en necropsias. Una revisión sistemática de estudios de necropsias no seleccionadas encontró $7,6 \%$ de carcinomas papilares y $0,14 \%$ carcinomas medulares, menores de $10 \mathrm{~mm}$ y no pesquisados en vida ${ }^{7}$. Contrastando con esta cifra, la incidencia de CT estimada mediante el registro de tumores con confirmación histopatológica SEER en Estados Unidos de Norteamérica fue 17,6 casos por 100.000 el año $2013^{3}$. Podemos suponer que esta tasa representa la frecuencia de una en- 
fermedad clínicamente relevante, sin embargo, $28,6 \%$ de los tumores medían menos de $10 \mathrm{~mm}$. Tampoco podemos asegurar que se incluyeron todos los casos que debieron ser operados.

El estudio INCATIR, en base a los registros histopatológicos de tiroidectomías, estimó que la incidencia de CT en Chile era 7,86 casos por 100.000 el año $2011^{6}$. Esta tasa está subestimada porque sólo $23 \%$ de las tiroidectomías fueron realizadas en hospitales de sistema público de salud cuando ese año en Chile el $81 \%$ de la población estaba afiliada al fondo nacional de salud (FONASA) que financia las prestaciones quirúrgicas en hospitales del sistema público ${ }^{8}$. En otras palabras, se produjo una subestimación por falta de acceso a la cirugía en las personas atendidas por el sector público.

Otro aspecto destacable es el cambio en las recomendaciones para el diagnóstico y tratamiento del nódulo tiroideo y del CT contenidas en la Guía Clínica del año 2015 de la American Thyroid Association (ATA) ${ }^{9}$ y que también se refleja en el Consenso de la Sociedad Chilena de Endocrinología y Diabetes (SOCHED) para el estudio y manejo de nódulos tiroideos por médicos no especialistas ${ }^{10}$. Nuestro propósito es conocer la incidencia de CT en una población chilena con acceso a la cirugía de tiroides. Como objetivo secundario queremos describir las características histopatológicas del CT operado con posterioridad a la comunicación de la Guía Clínica ATA 2015.

\section{Método}

Para obtener la población del estudio se seleccionó un centro de salud en Chile con alto flujo de cirugía de cabeza y cuello, con acceso expedito a diagnóstico y tratamiento, en que las decisiones terapéuticas se tomen en un comité y que atienda una población cautiva. El Instituto Oncológico Fundación Arturo López Pérez (FALP) reúne esas características. La población atendida en esta institución cuenta con la posibilidad de contrato de un seguro contra eventos neoplásicos, llamado convenio oncológico, que incluye la cobertura de todos los servicios de diagnóstico y tratamiento de un cáncer en la institución.

Por el efecto que las nuevas recomendaciones pudieran tener en el manejo del nódulo tiroideo y del $\mathrm{CT}^{9}$, fueron incluidas en este análisis todas las tiroidectomías realizadas entre el 1 de enero de
2016 y el 31 de diciembre de 2018 en la FALP. Luego de la aprobación del protocolo de investigación por el Comité de Ética de la institución, se tuvo acceso al registro de informes histopatológicos de todas las tiroidectomías realizadas en período señalado. Se incluyeron tiroidectomías totales, ampliadas y lobectomías tiroideas, se excluyeron disecciones ganglionares y paratiroidectomías sin resección de tejido tiroideo y las reoperaciones.

Se registraron edad, sexo, fecha de la cirugía y parámetros histopatológicos de valor pronóstico (tipo histológico, tamaño tumoral, multicentricidad, compromiso de bordes quirúrgicos, extensión extra-tiroidea, invasión vascular y compromiso ganglionar).

Para estimar la incidencia de CT se solicitó a la Unidad de Convenio oncológico de la FALP el número de personas afiliadas por año $(2016,2017$ y 2018) así como su distribución etaria, por sexo, previsión de salud y fecha de incorporación.

Para el análisis de los datos se utilizó el programa STATA 14.0, realizándose estadística descriptiva y pruebas de hipótesis para evaluar asociación de variables $\left(\chi^{2}\right.$ para variables discretas $y t$ test para variables continuas). Se consideró significativo un valor de p menor a 0,05. La incidencia de CT se estimó para cada año del período de estudio, utilizando en el denominador la población en convenio oncológico y en el numerador los casos de cáncer de pacientes en convenio, ya que en la FALP también son sometidas a cirugía de cabeza y cuello personas sin esta condición.

Para corregir los potenciales sesgos implícitos en proyectar la incidencia de cáncer de tiroides de la población en convenio oncológico FALP a la población chilena, se ajustó esta tasa por la distribución etaria y por sexo del censo $2017^{11}$. Para comparar con la incidencia comunicada por el estudio INCATIR ${ }^{6}$, se ajustó la tasa por la distribución etaria y por sexo del censo $2012^{12}$. En ambas situaciones se ajustó la población completa y sólo la mayor de 15 años. Todos los ajustes se realizaron utilizando el método directo.

\section{Resultados}

Durante el período 2016-2018 se realizaron 1.477 cirugías de tiroides en FALP, encontrándose neoplasia maligna en 1.058 casos $(71,63 \%)$ y de los cuales 991 tenían convenio oncológico. La pobla- 
ción en convenio era 545.364, 596.236 y 664.284 los años 2016, 2017 y 2018 respectivamente. La proporción de personas con previsión del Fondo Nacional de Salud (FONASA) fue 39,4\%, el promedio de permanencia en el convenio al momento de la cirugía fue 58,73 meses y la proporción de convenios con menos de un año de vigencia fue $9,38 \%$.

En la Figura 1 se puede apreciar la incidencia de CT por año y según sexo, destacando su gran magnitud especialmente en mujeres.

En la Figura 2 se muestran las diferencias entre la pirámide poblacional del convenio oncológico FALP y la población de Chile 2017. Al ajustar las tasas de incidencia de la población en convenio completa y sólo mayores de 15 años por la estructura poblacional chilena 2017 (Tabla 1) y por la estructura poblacional chilena 2012 (Tabla 2) se observan diferencias, pero las tasas persisten siendo notablemente altas.

La mayor frecuencia de CT se observó entre la cuarta y quinta década de la vida (Figura 3 ), no encontrando diferencia significativa en la edad promedio de varones y mujeres, que fueron 45,2 años (rango 21-79 años) y 44,3 años (rango 16-87 años) respectivamente. El $87 \%$ de los casos de CT ocurrieron en mujeres.

El tipo histológico más frecuente fue el carcinoma papilar (96,3\%), seguido por el carcinoma folicular (2,4\%) (Figura 4).

En la mayoría de los casos el tamaño tumoral fue menor de $20 \mathrm{~mm}$, siendo en promedio signi- ficativamente mayor en varones que en mujeres $(17,8 \mathrm{~mm}$ vs $13,6 \mathrm{~mm} ; \mathrm{p}<0,0001)$. La proporción de micro-tumores $(<10 \mathrm{~mm})$ fue $41 \%$ cuando se consideró el diámetro del nódulo dominante en tumores multicéntricos y $35,4 \%$ cuando se consideró la sumatoria de los diámetros de todos los focos (Figura 5). Setenta y cuatro de los 430 microtumores fueron casos incidentales de carcinoma papilar en tiroidectomías por hipertiroidismo o bocio multinodular, en que la indicación quirúrgica fue el tamaño o una citología indeterminada en otro nódulo. Si excluimos los casos incidentales del análisis la proporción de microtumores se reduce a $33,6 \%$. Estos microtumores fueron más frecuentes en carcinomas papilares que foliculares $(40 \%$ vs $10 \% ; \mathrm{p}<0,0001)$. No hubo casos de microtumor en los carcinomas medulares. La proporción de microtumores en varones fue significativamente más baja que en mujeres $(27,73 \%$ vs $42,45 \%$; $\mathrm{p}<0,001)$. En 28 de 356 (7,9\%) microtumores no incidentales se realizó lobectomía.

Al evaluar los criterios de extensión, 26,6\% de los casos de CT eran multicéntricos y $21,2 \%$ tenían invasión extratiroidea, sin diferencias significativas entre sexos. Sólo en $6 \%$ se observó compromiso de borde quirúrgico, siendo este hallazgo significativamente más frecuente en varones $(\mathrm{p}=0,028)$.

En 534 de 1.058 cirugías con hallazgo de CT se realizó resección ganglionar $(50,5 \%)$, encontrándose compromiso neoplásico en $63,67 \%$. El número de ganglios comprometidos en promedio fue significativamente mayor en varones que en

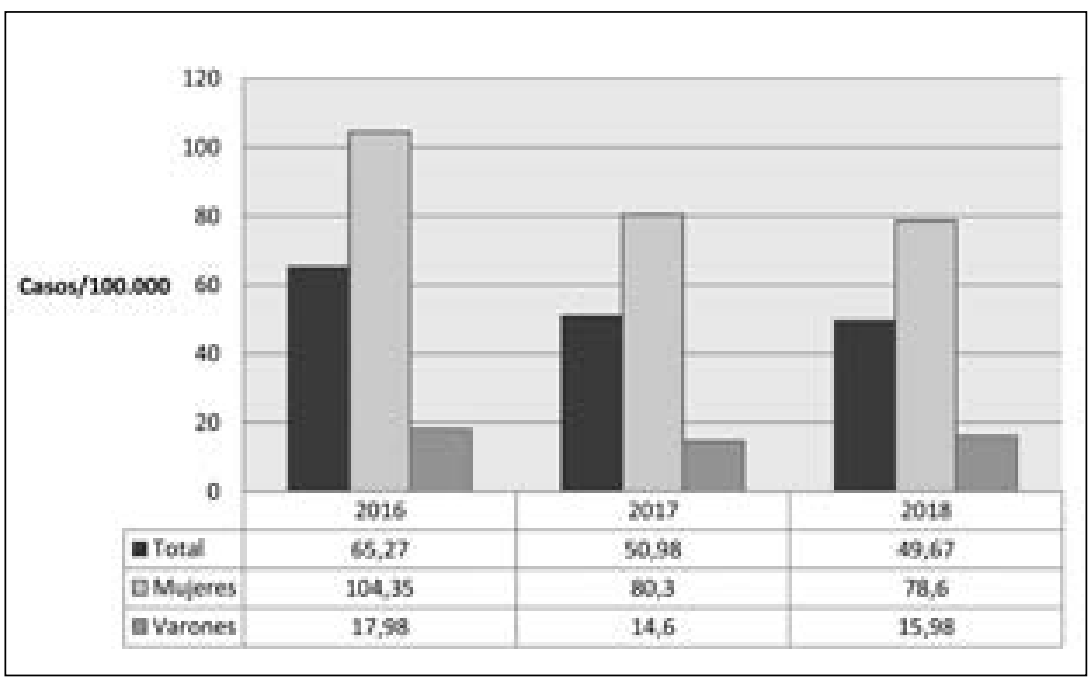

Figura 1. Incidencia de cáncer de tiroides en población en convenio oncológico FALP según año y sexo. 


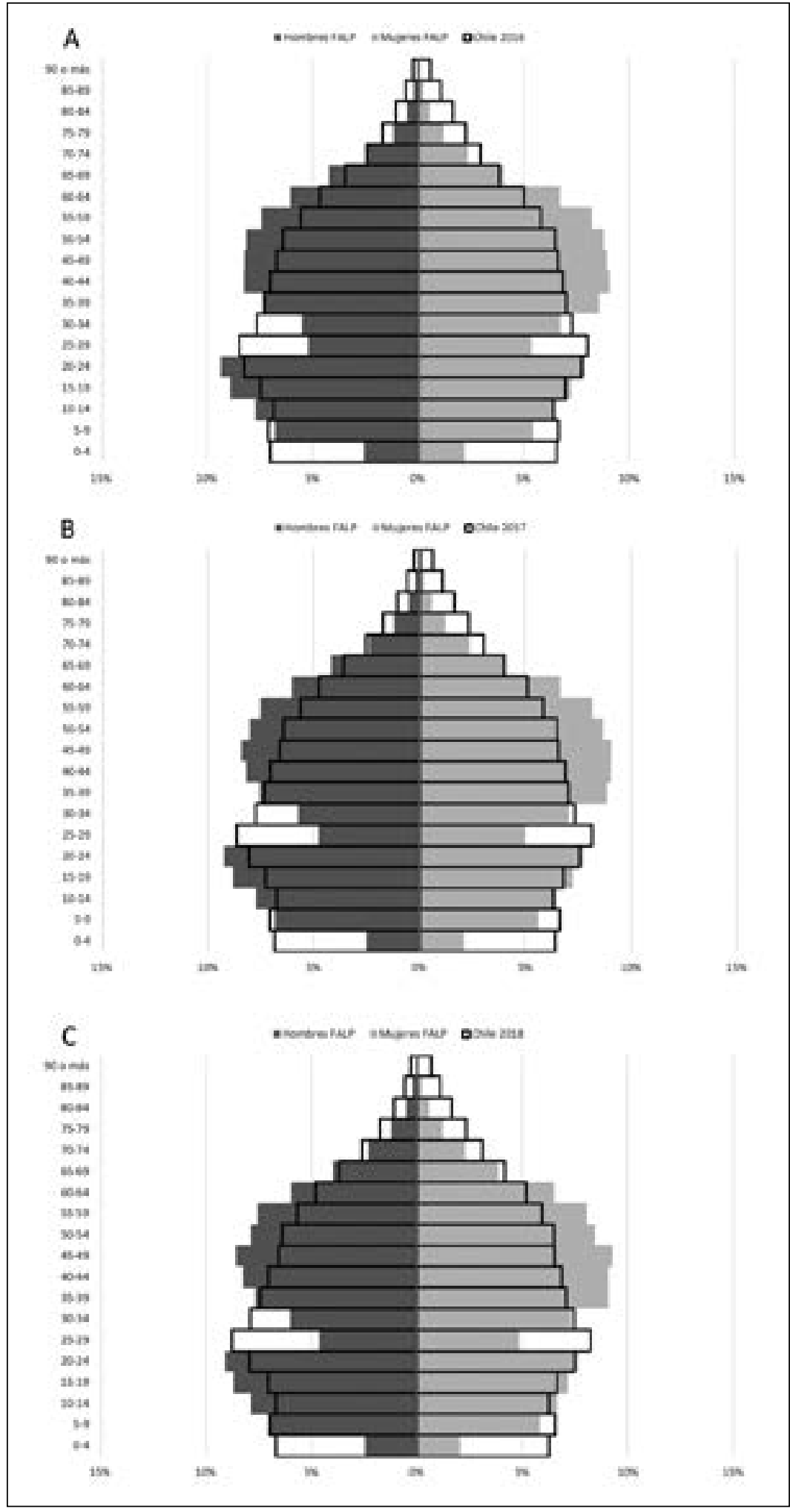

Figura 2. Pirámide poblacional de afiliados a convenio oncológico FALP los años 2016 (A), 2017 (B), 2018 (C) y población de Chile 2017. 
Epidemiología del cáncer de tiroides en Chile - J. Sapunar et al

Tabla 1. Incidencia de cáncer de tiroides en población en convenio oncológico faLP completa y sólo mayores de 15 años, por año, ajustada por la distribución etaria y por sexo de la población chilena año 2017

\begin{tabular}{|c|c|c|c|c|c|c|}
\hline \multirow{2}{*}{$\begin{array}{l}\text { Según Censo } 2017 \\
\text { Año }\end{array}$} & \multicolumn{3}{|c|}{15 o más (Tasa x 100.000) } & \multicolumn{3}{|c|}{ Población completa (Tasa x 100.000) } \\
\hline & Varones & Mujeres & Total & Hombres & Mujeres & Total \\
\hline \multicolumn{7}{|l|}{2016} \\
\hline No ajustada & 21,67 & 122,45 & 77,12 & 17,98 & 104,35 & 65,27 \\
\hline Ajustada & 20,84 & 119,52 & 76,25 & 16,46 & 96,03 & 60,75 \\
\hline \multicolumn{7}{|l|}{2017} \\
\hline No ajustada & 18,05 & 95,41 & 60,54 & 14,60 & 80,30 & 50,98 \\
\hline Ajustada & 19,72 & 93,20 & 60,58 & 15,64 & 75,14 & 48,44 \\
\hline \multicolumn{7}{|l|}{2018} \\
\hline No ajustada & 19,31 & 91,82 & 58,95 & 15,98 & 78,63 & 49,67 \\
\hline Ajustada & 21,03 & 95,11 & 62,09 & 16,33 & 75,24 & 48,66 \\
\hline
\end{tabular}

Tabla 2. Incidencia de cáncer de tiroides en población en convenio oncológico FALP, completa y sólo mayores de 15 años, por año, ajustada por la distribución etaria y por sexo de la población chilena año 2012

\begin{tabular}{|c|c|c|c|c|c|c|}
\hline \multirow{2}{*}{$\begin{array}{l}\text { Según Censo } 2012 \\
\text { Año }\end{array}$} & \multicolumn{3}{|c|}{15 o más (Tasa x 100.000) } & \multicolumn{3}{|c|}{ Población completa (Tasa x 100.000) } \\
\hline & Varones & Mujeres & Total & Hombres & Mujeres & Total \\
\hline \multicolumn{7}{|l|}{2016} \\
\hline No ajustada & 21,67 & 122,45 & 77,12 & 17,98 & 104,35 & 65,27 \\
\hline Ajustada & 20,41 & 118,30 & 75,29 & 15,82 & 93,46 & 58,93 \\
\hline \multicolumn{7}{|l|}{2017} \\
\hline No ajustada & 18,05 & 95,41 & 60,54 & 14,60 & 80,30 & 50,98 \\
\hline Ajustada & 19,23 & 92,00 & 59,55 & 14,91 & 72,68 & 46,61 \\
\hline \multicolumn{7}{|l|}{2018} \\
\hline No ajustada & 19,31 & 91,82 & 58,95 & 15,98 & 78,63 & 49,67 \\
\hline Ajustada & 20,51 & 93,64 & 60,95 & 15,90 & 73,97 & 47,71 \\
\hline
\end{tabular}

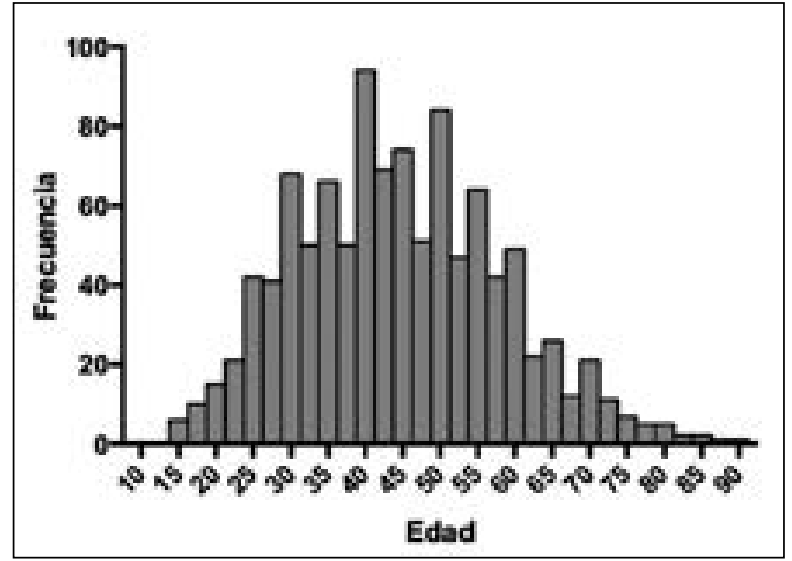

Figura 3. Distribución etaria del diagnóstico de cáncer de tiroides en pacientes operados en FALP entre los años 2016-2018 $(N=1.058)$.

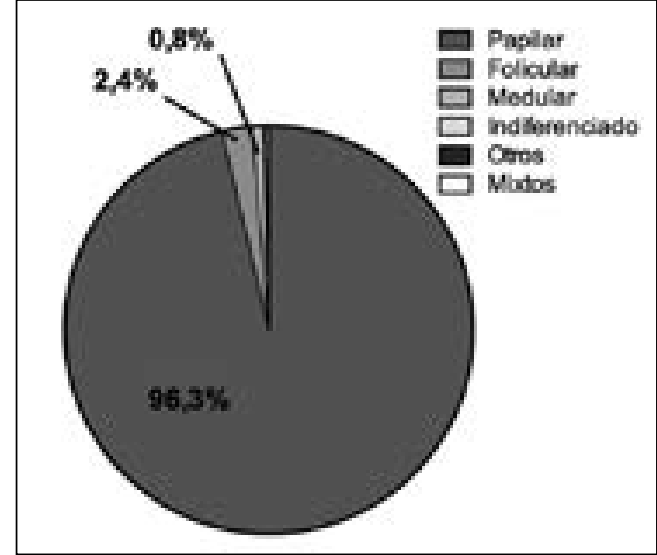

Figura 4. Distribución por tipo histológico de cáncer de tiroides en pacientes operados en FALP entre los años 2016-2018 ( $\mathrm{N}=1.058)$. 

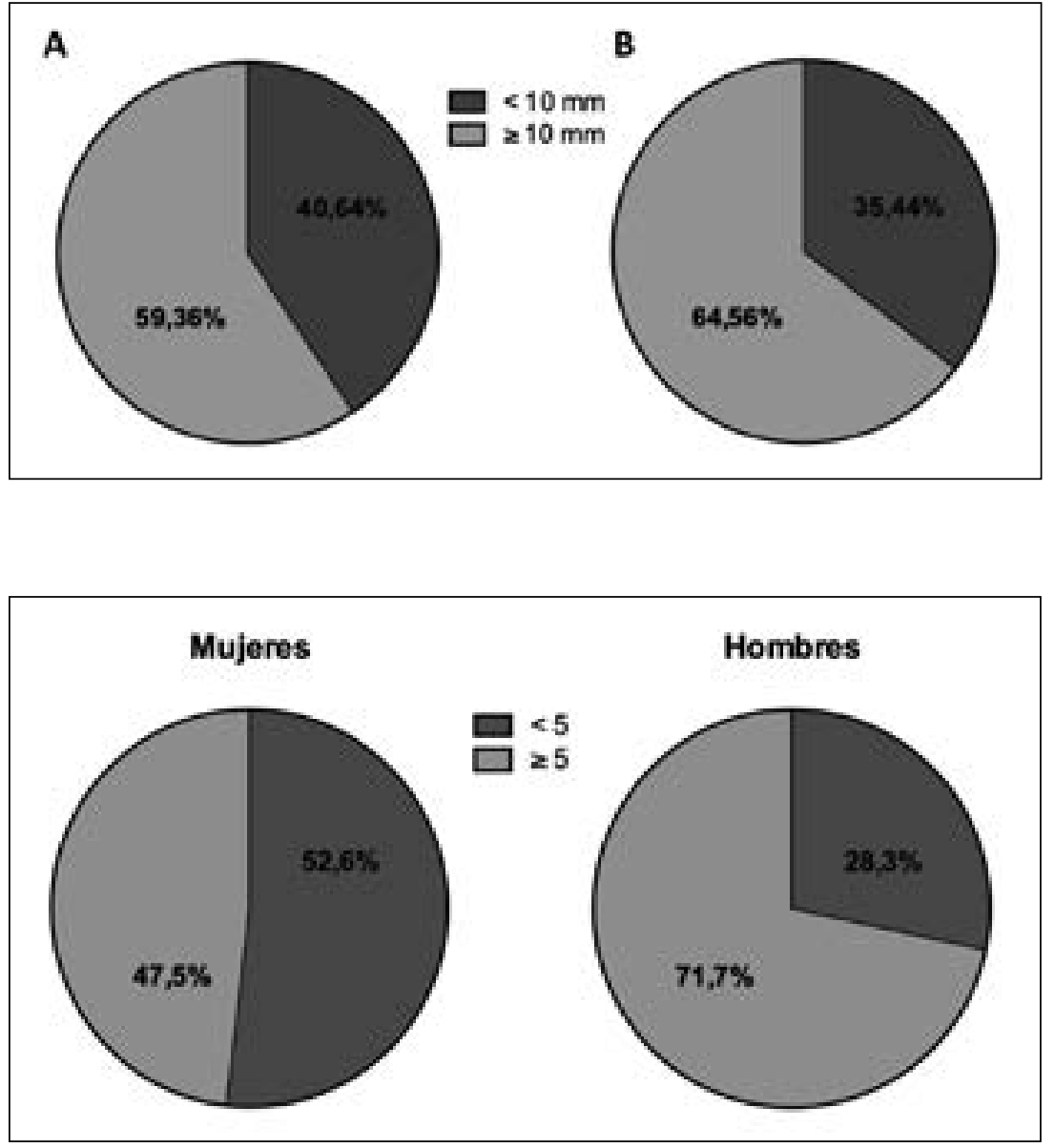

Figura 5. Distribución según categorías de tamaño tumoral, considerando el diámetro del foco dominante (A) o la sumatoria de diámetros (B) en cánceres multicéntricos en pacientes operados en FALP entre los años 2016-2018 ( $\mathrm{N}=1.058)$.
Figura 6. Proporción de pacientes con más de 5 ganglios comprometidos por cáncer de tiroides según sexo $(\mathrm{N}=1.058)$. mujeres ( $\mathrm{p}<0,0001)$. El 52,5\% de los pacientes en que se realizó resección ganglionar tenían más de 5 comprometidos. La proporción de pacientes con más de 5 ganglios comprometidos fue significativamente mayor en varones $(71,7 \%$ vs $47,5 \%$; $\mathrm{p}=0,005)$ (Figura 6).

El compromiso vascular no fue analizado por no estar descrito en todos los informes histopatológicos.

\section{Discusión}

El Departamento de Epidemiología del Ministerio de Salud (MINSAL) estimó que la incidencia de CT era 2 casos por 100.000 año en varones y 5,3 casos por 100.000 año en mujeres durante el período 2003-2007, en base a los datos registra- dos por 3 centros centinelas en las regiones de Antofagasta, Bío-Bío y Los Ríos 5 . Posteriormente el estudio INCATIR estimó la incidencia en 7,86 casos por 100.000 en población total, en base a los registros de informes histopatológicos de tiroidectomías realizadas en Chile durante el año 2011. Nuestro estudio reportó una incidencia de 60,75 casos por 100.000 el año 2016, 48,5 casos por 100.000 el año 2017 y 48,66 casos por 100.000 el año 2018, en base al registro de informes histopatológicos de un centro de salud con población cautiva. Este aparente aumento en la incidencia de CT en Chile, al comparar los resultados de estos 3 estudios, podría reflejar la tendencia que sigue la tasa a nivel mundial ${ }^{1}$, sin embargo, hay que considerar que la estimación de frecuencia mediante centros de monitoreo regional podría tener sesgos atribuibles a variabilidad geográfica y 
a la pérdida de casos por su resolución quirúrgica fuera de la red de salud regional. Por otra parte ya se mencionó que en el estudio INCATIR sólo el $23 \%$ de las tiroidectomías provenían de hospitales del sistema público de salud cuando el $81 \%$ de la población era beneficiaria del sistema público, sugiriendo un sesgo por falta de acceso a la cirugía.

Si comparamos la incidencia reportada por nuestro estudio con las tasas disponibles en la literatura, ésta sólo sería superada por la comunicada para Corea del Sur ${ }^{1,2,13}$. La mayoría de los estudios incluidos en las revisiones sistemáticas ${ }^{1,2}$ obtuvieron sus datos de registros de tumores y entregan tasas hasta el año 2013. La incidencia de 113 casos por 100.000 mujeres coreanas el año $2010^{13}$, fue atribuida a sobrediagnóstico y sobretratamiento como consecuencia de tamizaje con ecografía cervical ${ }^{14}$, sin embargo, si se excluyen del análisis los micro-tumores la incidencia en mujeres sería 83,47 casos por $100.000^{13}$. En Latinoamérica destacan la incidencia de CT del Ecuador, que fue estimada en 22 casos por 100.000 el año 2016, en base a registro de altas hospitalarias ${ }^{15}$ y la obtenida del registro de tumores de Sao Paulo, Brasil, que fue 18,43 casos por 100.000 año en mujeres y 4,42 casos por 100.000 año en varones en el período 1997-2008 ${ }^{16}$.

En todos los casos que se utilizan registros de tumores es posible que la incidencia sea subestimada en la medida que la población tenga más dificultades para acceder a la cirugía. En nuestro estudio es más importante considerar un sesgo de selección ya que se analizó la población de un convenio oncológico mayoritariamente adquirido por empresas para sus trabajadores, lo que representa una población aparentemente sana. Este sesgo se controla en parte porque la mayoría de los convenios son planes colectivos de empresas, evitando la autoselección. En la discusión de los resultados del estudio de $\mathrm{Lim}^{3}$ se menciona el efecto de campañas de tamizaje con ecotomografía tiroidea que realizan algunas empresas a sus empleados. Para la formalización del seguro oncológico es necesario aportar antecedentes médicos, lo que dificulta que ingresen personas con sospecha de enfermedad.

En relación a los cambios en las características histopatológicas del cáncer de tiroides que podrían asociarse a la aplicación de las recomendaciones de la Guía ATA $2015^{9}$, destacan la menor proporción de microtumores que encontramos en relación a lo comunicado en el estudio INCATIR para el año 2011 (33,6\% vs 48,5\%) . El segundo aspecto destacado es la mayor proporción de ganglios resecados comprometidos en nuestro estudio $(63,7 \%)$ en relación al estudio INCATIR $(41,5 \%$ en varones y $24,9 \%$ en mujeres), lo que podría reflejar mayor precisión quirúrgica por el uso sistemático de la ecografía de etapificación. La frecuencia de disecciones ganglionares en nuestra serie $(50,5 \%)$ fue mayor que la esperada en base a los hallazgos comunicados para la ecotomografía de etapificación ${ }^{9}$. Esta diferencia podría explicarse por las características de la enfermedad resecada más que a la realización de disecciones ganglionares profilácticas. A pesar de las recomendaciones de las guías clínicas la proporción de lobectomías de nuestra serie es baja, incluso en microtumores ${ }^{9}$.

Una de las peculiaridades de los estudios de incidencia de CT en Chile es la alta proporción de mujeres ( $87 \%$ población FALP y $85,3 \%$ estudio INCATIR), superior a la observada en otros países $^{1}$ y a lo esperado por la mayor tendencia de las mujeres chilenas a ocupar los servicios de salud ${ }^{17}$. La proporción de carcinomas papilares que encontramos $(96,3 \%)$ fue mayor aún que la comunicada en el estudio INCATIR $(91,8 \%)$, hecho que parece ser directamente proporcional a la incidencia del $\mathrm{CT}^{18}$.

Como en todos los estudios de descripción de hallazgos histopatológicos el CT en varones muestra características más desfavorables como mayor tamaño tumoral, mayor frecuencia de compromiso de borde quirúrgico, mayor frecuencia y número de ganglios comprometidos por el tumor.

La principal limitación de nuestro estudio es poder garantizar que no hubo sesgo en la población en convenio oncológico, sin embargo, este modelo permite controlar mejor el sesgo por no acceso a la salud que tienen los estudios en base a registros de tumores en países con cobertura de salud insuficiente.

Es importante establecer que factores de riesgo explican la alta y creciente incidencia de CT en nuestro país. En los últimos años se han asociado con mayor riesgo de CT, además de la exposición a radiación, la obesidad, el sedentarismo y la diabetes mellitus $2^{19-21}$, contaminantes químicos como bisfenol A y cadmio ${ }^{21,22}$, la baja ingesta de yodo y la ingesta excesiva de nitritos ${ }^{24,25} \mathrm{y}$ factores reproductivos en la mujer ${ }^{26}$.

En conclusión, la incidencia de CT en población chilena parece ser mucho más alta de lo esti- 
mado previamente por las limitaciones de acceso a la cirugía. Las recomendaciones de las últimas guías clínicas han reducido el número de microtumores operados y han mejorado el reconocimiento de ganglios comprometidos con tumor.

Agradecimientos: Por la colaboración de los médicos anatomo-patólogos Manuel Meneses y Ana María Carrasco en la obtención de los registros de histopatología y de la Sra. Daniela Madrid en la elaboración de las bases de datos.

\section{Referencias}

1. Wiltshire JJ, Drake TM, Uttley L, Balasubramanian SP. Systematic Review of Trends in the Incidence Rates of Thyroid Cancer. Thyroid 2016; 26: 1541-52.

2. La Vecchia C, Malvezzi M, Bosetti C, Garavello W, Bertuccio P, Levi F, Negri E. Thyroid cancer mortality and incidence: a global overview. Int J Cancer 2015; 136: 2187-95.

3. Lim H, Devesa SS, Sosa JA, Check D, Kitahara CM. Trends in Thyroid Cancer Incidence and Mortality in the United States, 1974-2013. JAMA 2017; 317: 1338-48.

4. https://www.minsal.cl/plan-auge-85/ (Acceso el 11 de marzo de 2020).

5. Unidad Vigilancia Enfermedades no Transmisibles y Estudios, Departamento de Epidemiología, Subsecretaría Salud Pública. Ministerio de Salud de Chile. Primer Informe Registros Nacionales de Cáncer de Chile. Quinquenio 2003-2007. http://epi.minsal.cl/ epi/0notransmisibles/cancer/INFORME\%20RPC\%20 CHILE\%2020032007,\%20UNIDAD\%20VENT,\%20 DEPTO.EPIDEMIOLOGIA-MINSAL,13.04.2012.pdf (Acceso el 18 de agosto de 2014).

6. Sapunar ZJ, Muñoz NS, Roa SJC. Estimation of thyroid cancer incidence in Chile based on pathological reports. Rev Med Chile 2014; (142): 1099-105.

7. Valle LA, Kloos RT. The Prevalence of Occult Medullary Thyroid Carcinoma at Autopsy. J Clin Endocrinol Metab 2011; 96: E109-13.

8. CASEN Módulo Salud 2011. http://observatorio.ministeriodesarrollosocial.gob.cl/layout/doc/casen/2013_ 03_27_Salud_CASEN_2011.pdf (Acceso el 11 de marzo de 2020).

9. Haugen BR, Alexander EK, Bible KC, Doherty GM, Mandel SJ, Nikiforov YE, et al. 2015. American Thyroid Association Management Guidelines for Adult Patients with Thyroid Nodules and Differentiated Thyroid Cancer: The American Thyroid Association Guidelines Task
Force on Thyroid Nodules and Differentiated Thyroid Cancer. Thyroid 2016; 26: 1-133.

10. Tala H, Díaz RE, Domínguez Ruiz-Tagle JM, Sapunar J, Pineda P, Arroyo Albala P, et al. Study and management of thyroid nodes by non specialist physicians: SOCHED consensus. Rev Med Chile 2017; 145: 1028-37.

11. http://resultados.censo2017.cl/ (Acceso el 11 de marzo de 2020).

12. http://www.iab.cl/wp-content/files_mf/resumencenso_2012.pdf (Acceso el 18 de agosto de 2014).

13. Kweon SS, Shin MH, Chung IJ, Kim YJ, Choi JS. Thyroid cancer is the most common cancer in women, based on the data from population-based cancer registries, South Korea. Jpn J Clin Oncol. 2013; 43 (10): 1039-46.

14. Ahn HS, Welch HG. South Korea's Thyroid-Cancer "Epidemic" Turning the tyde. N Engl J Med 2015; 373: 2389-90.

15. Salazar-Vega J, Ortiz-Prado E, Solis-Pazmino P, Gómez-Barreno L, Simbaña-Rivera K, Henríquez-Trujillo AR, et al. Thyroid Cancer in Ecuador, a 16years population-based analysis (2001-2016). BMC Cancer 2019; 19: 294. doi: 10.1186/s12885-019-5485-8.

16. Veiga LHS, Neta G, Aschebrook-Kilfoy B, Ron E, Devesa SS. Thyroid Cancer Incidence Patterns in Sao Paulo, Brazil and the US SEER Program 1997-2008. Thyroid 2013; 23: 750-7.

17. Departamento de Estadísticas e Información de Salud. Atenciones de la red asistencial. Resúmenes estadísticos mensuales 2016-2018. http://www.deis.cl/resúmenes-estadisticos-mensuales-deis/ (Acceso el 9 de marzo de 2020).

18. Aschebrook-Kilfoy B, Ward MH, Sabra MM, Devesa SS. Thyroid Cancer Incidence Patterns in the United States by Histologic Type, 1992-2006. Thyroid. 2011; 21: 12534.

19. Kitahara CM, Pfeiffer RM, Sosa JA, Shiels MS. Impact of overweight and obesity on U.S. papillary thyroid cancer incidence trends (1995-2015). J Natl Cancer Inst 2019. pii: djz202. doi: 10.1093/jnci/djz202.

20. Fiore M, Cristaldi A, Okatyeva V, Lo Bianco S, Oliveri Conti G, Zuccarello P, et al. Physical Activity and Thyroid Cancer Risk: A Case-Control Study in Catania (South Italy). Int J Environ Res Public Health 2019; 16 (8). pii: E1428. doi: 10.3390/ijerph16081428.

21. Aschebrook-Kilfoy B, Sabra MM, Brenner A, Moore SC, Ron E, Schatzkin A, et al. Diabetes and thyroid cancer risk in the National Institutes of Health-AARP Diet and Health Study. Thyroid 2011; 21: 957-63.

22. Li L, Ying Y, Zhang C, Wang W, Li Y, Feng Y, et al. Bisphenol A exposure and risk of thyroid nodules in 
Chinese women: A case-control study. Environ Int 2019; 126: $321-8$

23. Zhang Q, Jiang $\mathrm{C}$, Li H, Zhang $\mathrm{C}$, Wu H, Huang F. Effect of the Interaction Between Cadmium Exposure and CLOCK Gene Polymorphisms on Thyroid Cancer: a Case-Control Study in China. Biol Trace Elem Res 2019. doi: 10.1007/s12011-019-01904-2.

24. Zhang YL, Li P, Liu ZY, Yi JP, Chen Y, Zhang M, et al. Does relatively low iodine intake contribute to thyroid cancer? An ecological comparison of epidemiology. Me- dicine (Baltimore) 2019; 98 (41): e17539. doi: 10.1097/ MD.0000000000017539.

25. Aschebrook-Kilfoy B, Shu XO, Gao YT, Ji BT, Yang G, Li HL, et al. Thyroid cancer risk and dietary nitrate and nitrite intake in the Shanghai women's health study. Int J Cancer. 2013; 132: 897-904.

26. Mannathazhathu AS, George PS, Sudhakaran S, Vasudevan D, Krishna Km J, Booth C, et al. Reproductive factors and thyroid cancer risk: Meta-analysis. Head Neck 2019. doi: 10.1002/hed.25945. 